\title{
Electrokinetic Salt Removal from Porous Building Materials Using Ion Exchange Membranes
}

\author{
K. Kamran - M. van Soestbergen • L. Pel
}

Received: 7 February 2012 / Accepted: 3 October 2012

C) Springer Science+Business Media Dordrecht 2012

\begin{abstract}
The removal of salt from porous building materials under the influence of an applied voltage gradient normally results in high $\mathrm{pH}$ gradients due to the formation of protons and hydroxyl ions at the electrodes. The formed acidic and alkaline regions not only lead to disintegration of the porous material, but also affect the salt transport. In this work we use ion exchange membranes between the electrodes and the porous material to prevent the protons and hydroxyl ions from intruding into the material. The porous material used in this study is fired clay brick, which has been saturated with a $4 \mathrm{~mol} / \mathrm{l}$ sodium chloride solution prior to the desalination treatment. In order to experimentally determine the salt removal, we monitored the sodium ion concentration profiles across the material with nuclear magnetic resonance (NMR). In addition, we present theoretical predictions for the salt removal according to a model based on the Poisson-Nernst-Planck theory for ion transport. From the work reported here, we can conclude that the use of ion exchange membranes to desalinate porous building materials is not useful since it reduces the salt removal rate to such an extent that desalination with poultices, which is driven by diffusion only, is more efficient. The reason behind this is twofold. First, the ion exchange membranes provide a penalty for the ions to leave the material. Second, in the absence of acidic and alkaline regions, the salt concentration at the edges of the porous material will reduce to almost zero, which leads to a locally increased electrical resistance, and thus a reduction of the electrical field in the bulk of the material. Due to this reduction the effect of the applied voltage gradient across the material vanishes, and the salt removal is limited by diffusion.
\end{abstract}

Keywords Electrokinetic remediation - Fired clay brick - Ion exchange membranes . Nuclear magnetic resonance $\cdot$ Desalination

K. Kamran · M. van Soestbergen · L. Pel $(\varangle)$

Department of Applied Physics, Eindhoven University of Technology,

P.O. Box 513 5600, MB, Eindhoven, The Netherlands

e-mail: L.pel@tue.nl

M. van Soestbergen

Materials innovation institute, Mekelweg 2, CD2628, Delft, The Netherlands 


\section{Nomenclature}

$B_{0}$

$C^{*}$

$C_{\mathrm{i}}$

$D$

$D^{*}$

F

$f_{1}$

I

$J$

$L$

$R$

$T$

$T$

V

$x$

$z$

$\Delta V_{\mathrm{D}}$

$\rho$

$\kappa$

$\eta$

$\mu$

$\gamma$

$\rho_{\mathrm{M}}$

$\tau$

\section{Sub- and Superscripts}

$a q$
$\mathrm{Cl}$
$\mathrm{H}$
$\mathrm{M}$
$\mathrm{Na}$
$\mathrm{OH}$
0

Static magnetic field (T)

Dimensionless ion concentration

Ion concentration $\left(\mathrm{mol} / \mathrm{m}^{3}\right)$

Diffusion coefficient $\left(\mathrm{m}^{2} / \mathrm{s}\right)$

Ambipolar diffusivity $\left(\mathrm{m}^{2} / \mathrm{s}\right)$

Faraday constant $(\mathrm{C} / \mathrm{mol})$

Larmor frequency $(\mathrm{MHz})$

Electrical current density $\left(\mathrm{A} / \mathrm{m}^{2}\right)$

Ion flux $\left(\mathrm{mol} /\left(\mathrm{m}^{2} \mathrm{~s}\right)\right)$

Sample length (m)

Gas constant $(\mathrm{J} /(\mathrm{mol} \mathrm{K}))$

Temperature (K)

Time (s)

Voltage (Volt)

Spatial coordinate $(\mathrm{m})$

Valence number

Donnan potential (Volt)

Dimensionless space charge density

Electrolyte conductivity (S/m)

Desalination efficiency (\%)

Ionic mobility $\left(\mathrm{m}^{2} /(\mathrm{sV})\right)$

Gyromagnetic ratio $(\mathrm{MHz} / \mathrm{T})$

Ion exchange capacity (meq/g)

Tortuosity

Aqueous solution

Chloride

Protons

Membrane

Sodium

Hydroxyl ions

Initial condition

\section{Introduction}

In this paper we report on electrokinetic desalination of porous building materials using ion exchange membranes. These membranes suppress the ingress of protons and hydroxyl ions, which create acidic and alkaline conditions. Electrokinetic desalination aims to remove salt ions from porous materials by the application of a voltage gradient between electrodes. The applied voltage gradient between the electrodes will result in the transport of ions towards the electrodes, where they accumulate. A deficit of ions in the material, which results in desalination, occurs when the amount of this accumulation is sufficiently large, or when the ions are continuously removed from the electrodes by flushing with water.

In this section we first discuss three application areas of electrokinetic desalination. Next, we report on the use of ion exchange membranes to improve the desalination efficiency. Finally, we will briefly outline the paper. 


\subsection{Desalination of Water}

Electrokinetic desalination can, e.g., be used to remove salt from water flowing through micro-channels by placing porous carbon electrodes, which have a high ion storage capacity, adjacent to these channels (Johnson and Newman 1971; Farmer et al. 1996; Zhao et al. 2010). It was demonstrated that it is possible to obtain potable water from brackish water using this method (Zhao et al. 2010). In addition, a very recent paper describes the possibility of removing salt from water by dipping an array of porous carbon-coated wires in water (Porada 2012). During this process ions from the salt water adsorb electrokinetically into the porous material and are released again after dipping the array in the brine.

\subsection{Decontamination of Soils}

Another area of interest for electrokinetic desalination is the removal of (toxic) ions from contaminated soils (Yeung and Gu 2011). Here metal electrodes are placed within the soil, meters apart, and a high electrode voltage is applied, while the toxic ions are removed by flushing the electrodes with water. Performing both laboratory experiments and field tests Probstein and Hicks (1993) showed that (heavy) metal ions can be removed from soils by electrokinetics. Later Ribeiro and Mexia (1997) studied copper contaminated soil from a wood preservation site. They clamped samples of this soil between two electrode compartments and found that the amount of copper removal was time-dependent and inhomogeneous across the sample. Yang and Lin (1998) used silt loam soil that was intentionally contaminated with lead, and found that the $\mathrm{pH}$ of both the soil and the electrode compartments are important for the removal efficiency. They concluded that more research was needed to optimize these parameters. Viadero et al. (1998) studied the $\mathrm{pH}$ of the soil more thoroughly and found that the critical $\mathrm{pH}$ to ensure effective lead removal lies between 4 and 4.5. Subsequently, Ottosen et al. (2000) used ion exchange membranes at the electrodes to control the $\mathrm{pH}$ within the soil (see Sect. 1.4). Using this method they could acidify the material, which increased the dissolution of copper ions, and thus enhanced their removal. Ottosen and co-workers also used their electrokinetic setup containing ion exchange membranes to decontaminate harbor sediment (Nystrøm, et al. 2005). They found removal efficiencies up to $94 \%$ for cadmium, $91 \%$ for zinc, and $73 \%$ for copper while stirring the sediment. However, precipitation of metal hydroxides remained a problem. In addition, Kim and Kim (2001) concluded that the removal efficiency depends on the affinities of the metal ions in the soil. Namely, they found that the efficiencies of mobile ions, such as cadmium, copper, and zinc, were higher than those of less mobile ions, such as lead.

\subsection{Electrokinetic Salt Removal from Building Materials}

Yet another application area is the removal of salt from historic building materials to prevent them from salt-induced decay. (Ottosen and Rörig-Dalgaard 2009; Kamran et al. 2012a). Salt-induced decay is the result of stress build-up in porous materials due to dissolution/crystallization cycles inside their porous structure, and can lead to severe disintegration. The disintegration of porous building materials and structures by water-soluble salts is a wellknown phenomenon with significant implications, since it can destroy objects of cultural heritage. In order to identify crystallization locations within porous materials Sghaier et al. (2007) developed a model for ion transport during drying. They found that the ion concentration during drying strongly depends on the effective ion diffusion coefficient, which is a function of the water saturation level in the porous material. The effective salt diffusion 
coefficient in a brick sample was determined experimentally by Lu and Viljanen (2002), who applied a salt concentration difference across the material, and found an effective diffusion coefficient of $3.5 \times 10^{-10} \mathrm{~m}^{2} / \mathrm{s}$. Kamran et al. (2012a) found a diffusion coefficient equal to $8 \times 10^{-10} \mathrm{~m}^{2} / \mathrm{s}$ for a corresponding brick material by measuring the salt removal with NMR. In cement paste, however, a simple diffusion coefficient might not be sufficient to describe the transport of ions since Coussy and Eymard (2003) found that chloride adsorbs onto the internal surface area of these materials. For a laboratory experiment on building materials Ottosen and Rörig-Dalgaard (2009) achieved a $99 \%$ salt removal efficiency within 9 days using electrokinetic desalination. Here they found a higher removal rate for $\mathrm{KCl}$ than for $\mathrm{NaCl}$, which was explained by the higher diffusion coefficient of $\mathrm{KCl}$ compared to $\mathrm{NaCl}$.

\subsection{Ion Exchange Membranes}

Due to the high electrode voltages used in the latter two application areas (soil and building materials), water splitting can be a major issue. The splitting of water can result in large pH gradients throughout the material (Probstein and Hicks 1993; Ribeiro and Mexia 1997; Ottosen et al. 2000; Kamran et al. 2012b), which have negative side effects. An acidic environment (i.e., a low pH) can, e.g., induce corrosion in reinforced concrete (Beddoe and Dorner 2005), or damage the mortar in masonry structures (Ottosen and Rörig-Dalgaard 2009), while in an alkaline environment (i.e., a high $\mathrm{pH}$ ) ions might immobilize and insoluble hydroxyl complexes can be formed (Probstein and Hicks 1993; Jacobs et al. 1994). In addition, acidic or alkaline conditions can attack the pigments in frescoes, which leads to an undesirable change of color of these objects of cultural heritage.

In order to suppress the ingress of acid and alkaline fronts the use of ion exchange membranes has been reported (Ribeiro and Mexia 1997; Ottosen et al. 2000; Nystrøm, et al. 2005; Yeung and $\mathrm{Gu}$ 2011). These membranes discriminate between the passages of positively and negatively charged ions, resulting in a permeselective barrier. Therefore, researchers have used membranes that only allow the passage of anions, i.e., anion exchange membranes, near the positively charged electrode, where an acidic environment prevails, to block the $\mathrm{H}$ ions from intruding into the material. Cation exchange membranes are used to block $\mathrm{OH}$ ions at the negative electrode, since here we have an alkaline environment. In soil the reported improvement in desalination performance is mainly due to the reduction of the alkaline region in which (toxic) metal ion hydroxyl complexes can get trapped (Probstein and Hicks 1993; Jacobs et al. 1994).

\subsection{Outline}

In porous building materials, which we study in this work, the main harmful contaminants are salts that do not form insoluble hydroxyl complexes, such as $\mathrm{NaCl}$. Therefore, we will study whether an improvement in desalination performance, similar to that achieved in soils, can be achieved in building materials using ion exchange membranes. In addition, we will study the efficiency of the membranes to block protons and hydroxyl ions, and thus the formation of acidic and alkaline conditions. In order to experimentally monitor the electrokinetic desalination of porous building materials we use nuclear magnetic resonance (NMR). In order to do this we analyze the removal of $\mathrm{NaCl}$ from cylindrical fired clay brick samples that are initially saturated with a $4 \mathrm{~mol} / \mathrm{l} \mathrm{NaCl}$ solution. Our NMR setup provides a unique opportunity to precisely monitor the evolution of the Na concentration profiles non-destructively with a high spatial resolution. To the best of our knowledge, we are not aware of a previous 
(non-destructive) experimental treatment with comparable accuracy that considers electrokinetic desalination of porous building materials using ion exchange membranes.

This paper is organized as follows. We will first present a theoretical study based on the Poisson-Nernst-Planck theory for ion transport. The emphasis is hereby on electrokinetic desalination in the presence of ion exchange membranes. We will then describe the experimental setup. Finally, we will present both the experimental and model results for the $\mathrm{Na}$ ion concentration profiles.

\section{Theoretical Section}

In this section we present an one-dimensional model with spatial coordinate $x$. We will analyze a cylindrical sample, which is sandwiched between a stack of wetted sponges and the ion exchange membranes. This system reflects the experimental setup as given in Fig. 1. Here the sponges provide a good contact between the membranes and the porous material. Near the positively biased electrode (anode) we use an anion exchange membrane to prevent the $\mathrm{H}$ ions that are formed at the anode to enter the material. Similarly, we use a cation exchange membrane near the negative electrode to prevent the $\mathrm{OH}$ ions from entering the porous material. Both membranes are continuously flushed with tap water to remove any ions that cross a membrane.

The transport of ionic species in a dilute electrolyte can in general be described by the Nernst-Planck equation (Newman 1973). In the absence of advection of the solvent and chemical reactions within the electrolyte, we can substitute the Nernst-Planck flux equation into a mass balance to obtain,

$$
\frac{\partial C_{i}}{\partial t}=-\frac{\partial J_{i}}{\partial x}=\frac{\partial}{\partial x}\left[\frac{1}{\tau}\left(D_{i} \frac{\partial C_{i}}{\partial x}+z_{i} \mu_{i} C_{i} \frac{\partial V}{\partial x}\right)\right]
$$

where $\partial C_{i} / \partial t$ is the time-derivative of the ion concentration $i, J_{i}$ is their flux, $\tau$ is the tortuosity, $D_{i}$ is the diffusion coefficient, $z_{i}$ is the valence, $\mu_{i}$ is the ionic mobility, and $V$ is

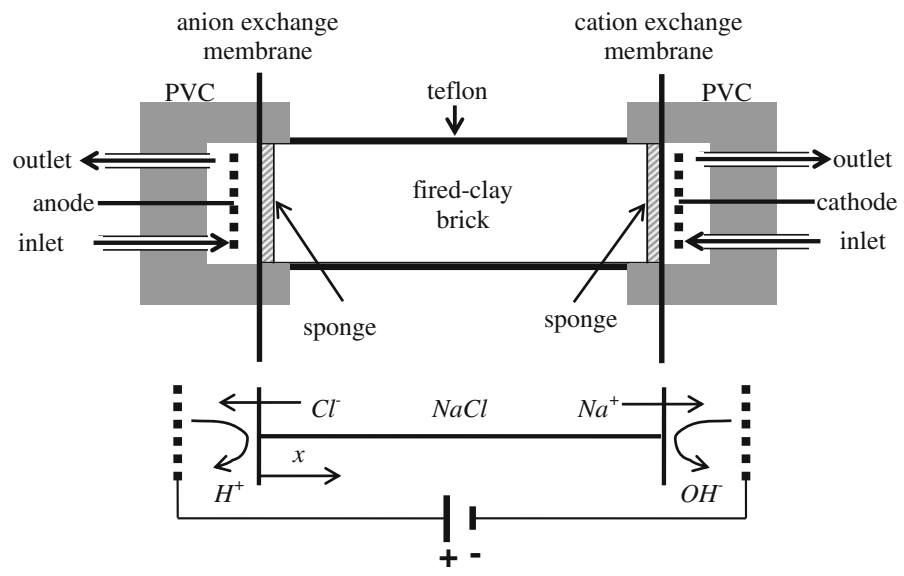

Fig. 1 A schematics representation of the experimental setup (top) and the theoretical model (bottom). An initially NaCl-saturated fired clay brick sample is sandwiched between two membranes, which either allow the $\mathrm{Cl}$ or $\mathrm{Na}$ ions to enter the anode or cathode compartment. Both compartments are flushed with tap water, while a voltage is applied between the electrodes 
the local voltage. In the system considered here we have two ionic species, namely monovalent chloride anions, $\mathrm{Cl}$, and monovalent sodium cations, $\mathrm{Na}$. Below we will simplify the mathematical framework and define the boundary conditions.

In order to simplify the governing equations we can define a dimensionless salt concentration as:

$$
C^{*}=\left(C_{\mathrm{Na}}+C_{\mathrm{Cl}}\right) /\left(C_{\mathrm{Na}}^{0}+C_{\mathrm{Cl}}^{0}\right),
$$

and the dimensionless space charge density as:

$$
\rho=\left(C_{\mathrm{Na}}-C_{\mathrm{Cl}}\right) /\left(C_{\mathrm{Na}}^{0}+C_{\mathrm{Cl}}^{0}\right),
$$

where superscript 0 denotes the initial ion concentration and subscript $\mathrm{Na}$ and $\mathrm{Cl}$ indicate the sodium and chloride ions, respectively. Next we will assume electro-neutrality, i.e., $C_{\mathrm{Cl}}=$ $C_{\mathrm{Na}}$ (and hence $\rho=0$ ), through most the porous material, which is common in the field of electrochemistry (Newman 1973). In addition, we use the fact that the regions containing a considerable non-zero space charge density are confined to nanoscopic layers near the edges of the system (Smyrl and Newman 1967; Bonnefont et al. 2001; Bazant et al. 2004; Prieve 2004; Bazant et al. 2005; Biesheuvel et al. 2009a,b; van Soestbergen 2010; van Soestbergen et al. 2010; Chu and Bazant 2005); We can describe these regions, the so-called diffuse layers, using their equilibrium properties since their dynamics is much faster than the bulk transport (Smyrl and Newman 1967; Bazant et al. 2004; Prieve 2004; Bonnefont et al. 2001; Bazant et al. 2005; Biesheuvel et al. 2009a,b; van Soestbergen 2010; van Soestbergen et al. 2010; Chu and Bazant 2005), and incorporate them into the boundary conditions.

Substitution of the mass balance of Eq. (1) in both the time-derivatives of Eqs. (2) and (3) while using electro-neutrality, i.e., $C_{\mathrm{Na}}=C_{\mathrm{Cl}}=\frac{1}{2} C^{*}\left(C_{\mathrm{Na}}^{0}+C_{\mathrm{Cl}}^{0}\right)$, results in,

$$
\frac{\partial C^{*}}{\partial t}=\frac{1}{2 \tau} \frac{\partial}{\partial x}\left[\left(\left(D_{\mathrm{Na}}+D_{\mathrm{Cl}}\right) \frac{\partial C^{*}}{\partial x}+\left(\mu_{\mathrm{Na}}-\mu_{\mathrm{Cl}}\right) C^{*} \frac{\partial V}{\partial x}\right)\right],
$$

for the mass balance of the dimensionless salt concentration, and

$$
\frac{\partial \rho}{\partial t}=\frac{1}{2 \tau} \frac{\partial}{\partial x}\left[\left(\left(D_{\mathrm{Na}}-D_{\mathrm{Cl}}\right) \frac{\partial C^{*}}{\partial x}+\left(\mu_{\mathrm{Na}}+\mu_{\mathrm{Cl}}\right) C^{*} \frac{\partial V}{\partial x}\right)\right],
$$

for the charge balance of the dimensionless space charge density.

Again applying the electro-neutrality assumption results in $\partial \rho / \partial t=0$. Consequently, we can obtain an expression for the voltage distribution from Eq. (5),

$$
\frac{\partial}{\partial x}\left(C^{*} \frac{\partial V}{\partial x}\right)=-\frac{D_{\mathrm{Na}}-D_{\mathrm{Cl}}}{\mu_{\mathrm{Na}}+\mu_{\mathrm{Cl}}} \frac{\partial^{2} C^{*}}{\partial x^{2}} .
$$

In case we neglect any redistribution of species in the material, the latter equation follows Ohm's law where the electrical current density is given by $I=\kappa \partial V / \partial x$, with the electrolyte conductivity, $\kappa=F C^{*}\left(\mu_{\mathrm{Na}}+\mu_{\mathrm{Cl}}\right) / 2 \tau$, where $F$ is Faraday's constant. Therefore, we can consider Eq. (6) as a modified Ohm's law that accounts for ion redistribution. Substitution of Eq. (6) in (4) gives (Levich 1962; Newman 1973; Vetter 1967; Bard and Faulkner 2001; Bazant et al. 2004);

$$
\frac{\partial C^{*}}{\partial t}=D^{*} \frac{\partial^{2} C^{*}}{\partial x^{2}}
$$

where $D^{*}=\tau^{-1}\left(\mu_{\mathrm{Cl}} D_{\mathrm{Na}}+\mu_{\mathrm{Na}} D_{\mathrm{Cl}}\right) /\left(\mu_{\mathrm{Cl}}+\mu_{\mathrm{Na}}\right)$, which is the "ambipolar diffusivity" of the salt. The latter equation, Eq. (7), resembles Fick's second law for the diffusion of 
neutral species (Crank 1990; Deen 1998), and thus indicates that the hydrated $\mathrm{Na}$ and $\mathrm{Cl}$ ions diffuse through the porous material as conjugated particles due to the high electrostatic forces required to separate them.

The boundary conditions for Eq. (7) are determined by the membranes. Within the polymeric ion exchange membranes we have a high concentration of polarized groups that are immobile, which results in an equivalent high charge density that is trapped within the material. This charge density is countered by oppositely charged mobile ions, i.e., the counterions. These ions can thus penetrate through the membrane from the aqueous solution in which the membrane is submerged. As a result, the concentration of counterions is much higher than the concentration of co-ions, i.e., ions having the same charge sign as the polarized groups in the material. For a single salt consisting of only one species of co- and counterions, the counterion concentration is ideally constant across the membrane and the diffusive flux is thus negligible. Consequently, the migration of counterions through the membrane is much higher than that of co-ions and the membranes preferentially allow the transport of counterions.

In the absence of an applied voltage gradient across the membrane, the ions can still penetrate through the membrane from a reservoir with a high counterion concentration to a reservoir with a low counterion concentration due to concentration polarization at the membrane-reservoir interfaces. This polarization results from the difference in ion concentration within the membrane and the aqueous solution surrounding it. In our system the concentrations within the membrane are such that $C_{\mathrm{Na}}-C_{\mathrm{Cl}}+\rho_{\mathrm{M}} \approx 0$ and in the aqueous solution we have $C_{\mathrm{Na}}-C_{\mathrm{Cl}} \approx 0$, where $\rho_{\mathrm{M}}$ is the equivalent charge density in the membrane, i.e., the ion exchange capacity (Dlugolecki et al. 2008). The difference in ion concentration between the membrane and the aqueous solution results in a thin layer of diffuse charge at the membrane surface (polarization) with a voltage drop (Biesheuvel and van der Wal 2010; Nikonenko et al. 2010);

$$
\left.C_{i}\right|_{M}=\left.C_{i}\right|_{\mathrm{aq}} \exp \left[-\frac{z_{i} F}{R T} \Delta V_{D}\right]
$$

where $\Delta V_{D}$ is the voltage drop across the diffuse charge layer, i.e., the so-called Donnan potential, $F$ the Faraday constant, $R$ the gas constant, $T$ the temperature, and subscript $M$ and aq denote the concentration within the membrane and the aqueous solution, respectively. When the ion concentrations at both sides of the membranes are unequal the Donnan potentials at both sides of the membrane are unequal too. Therefore, we can have a voltage drop across the membrane for unequal ion concentrations at both sides of the membrane, even if the applied voltage gradient between both these aqueous phases equals zero. In our system we use tap water with a typical concentration of $\mathrm{NaCl} 10 \mathrm{mM}$ (Zhao et al. 2010) at one side of the membrane while the concentration at the other side is initially $\sim 4000 \mathrm{mM}$. This results in a voltage drop across the membrane of $\sim 0.1$ Volt, while the resistance of our membranes with an area of $1 \mathrm{~cm}^{2}$ is in the order of $1-3 \Omega$ (Dlugolecki et al. 2008). Consequently, we can have a flux of ions equivalent to an electrical current in the order of $100 \mathrm{~mA}$, which is of the same order as found for electrokinetic desalination in the absence of membranes (Kamran et al. 2012a). As a result, the ions that are at the edge of the sample will readily pass through the membrane due to the high difference in ion concentration across the membrane.

We can only determine the total voltage drop across the whole system by taking into account the above-mentioned polarization of the membrane surface and the electrodes. However, this is not straightforward since a mathematical description of the polarization of the electrodes at the high electrode voltages that we apply is ambiguous. Therefore, we will not use the applied voltage gradient between the electrodes as the input parameter, but imply an 
electrical current instead. We can simply determine this current as function of time experimentally and feed it into the model.

The applied electrical current through the system must be conserved since we cannot store charge in the material. Consequently, we obtain the following boundary conditions at the edges of the porous material,

$$
D^{*} \frac{\partial C^{*}}{\partial x}=-\left(J_{\mathrm{Na}}+J_{\mathrm{Cl}}\right)= \pm \frac{I}{F\left(C_{\mathrm{Na}}^{0}+C_{\mathrm{Cl}}^{0}\right)},
$$

where the \pm sign refers to the positive value at $x=0$ and the negative value at $x=L$, with $L$ the length of the material. Note that this boundary condition originates from the membranes blocking the Na flux at $x=0$ and the $\mathrm{Cl}$ flux at $x=L$. The effects of the applied voltage and the ion exchange membranes are now incorporated in the electrical current, which we can determine experimentally. Consequently the time-dependent salt profiles across the porous material can be calculated by solving the Fickian diffusion equation, i.e., Eq. (7), with the boundary condition of Eq. (9).

\section{Experimental Section}

The samples used in this study were cylindrical fired clay bricks with a length of 90 and $20 \mathrm{~mm}$ diameter, which were drilled from bricks. We use fired clay brick since its inert nature makes it a suitable material to be studied and it is also commonly used in many historical buildings. Prior to vacuum saturating the samples with a $4 \mathrm{M} \mathrm{NaCl}$ solution at room temperature, they were dried at $105{ }^{\circ} \mathrm{C}$ for $24 \mathrm{~h}$. Though this $4 \mathrm{M}$ concentration might appear unrealistic, it can occur in bricks where salt accumulates due to cyclic loading over a longer period. During the desalination experiments, the longitudinal surfaces of the samples were wrapped in Teflon tape to avoid drying. The experimental setup consists of two PVC sample holders that encapsulate the specimen at both sides (see Fig. 1). Within these sample holders either a cation or anion membrane (Neosepta CMX or AMX, Tokuyama Co., Japan) is clamped. In addition, some grease is used at the membrane-PVC interfaces to avoid water leakage. The cation exchange membrane has an ion exchange capacity of 1.62 meq./g, a permselectivity of $>96 \%$, an electrical resistance of $2.9 \Omega \mathrm{cm}^{2}$, and a thickness of $160 \mu \mathrm{m}$, while these properties for the anion exchange membrane are 1.25 meq./g, $>91 \%, 2.4 \Omega \mathrm{cm}^{2}$, and $134 \mu \mathrm{m}$, respectively (Dlugolecki et al. 2008). Through the cavities in the holders we flow tap water at approximately $5 \mathrm{ml} / \mathrm{min}$. Note that the water permeability of the membranes is sufficiently low to marginalize salt transport by hydraulic advection. The outlet of the sample holder where we apply a positive voltage is connected to the inlet where we apply the negative voltage to neutralize the water flow as much as possible. Namely, water splitting at the electrodes will either introduce $\mathrm{H}$ or $\mathrm{OH}$ ions, depending on their voltage, which will result in an acidic or alkaline environment, respectively. The electrodes are inserted in the cavity as circular wound platinum wires. An electrical voltage gradient is applied across the specimen using a DC power supply (Delta Electronika SM70-AR-24), while the current is monitored by a digital multi-meter (Dynatek D9200). In order to provide a good contact we apply sponges between the membrane and the porous sample. These sponges are pre-wetted to prevent the edges of the sample from drying.

The NMR setup we use for non-destructively monitoring the $\mathrm{H}$ and Na profiles across the specimen has been extensively described in Kamran et al. (2012a). In brief, NMR exploits the magnetic properties of nuclei possessing non-zero magnetic moments. When such nuclei are placed in an externally applied static magnetic field, $B_{0}$, their magnetic moments precess 
around this field. The resonance frequency of these nuclei is given by the Larmor frequency (Abragam 1961), $f_{l}=\gamma_{i} B_{0}$, where subscript $i$ refers to the type of nucleus ( $\mathrm{H}$ or $\mathrm{Na}$ in this work) and $\gamma_{i}$ is the gyromagnetic ratio, $\left(\gamma_{\mathrm{H}}=42.58 \mathrm{MHz} \mathrm{T}^{-1} ; \gamma_{N a}=11.27 \mathrm{MHz} \mathrm{T}^{-1}\right)$. We record the NMR signals according to the so-called Hahn-spin-echo sequence (Hahn 1950) for both $\mathrm{H}$ and $\mathrm{Na}$. This is done by switching between the radio frequencies that correspond to their Larmor frequencies with a specially designed RF circuit (Pel et al. 2000). Consequently, the recorded signal is proportional to the density of the nuclei under investigation (Abragam 1961). The NMR signal only represents dissolved $\mathrm{Na}$ ions and does not capture any salt crystals. Due to its low NMR sensitivity, $\mathrm{Cl}$ is not considered in this study. In our NMR setup we use a conventional electromagnet that produces a constant magnetic field of $0.96 \mathrm{~T}$. We use a magnetic field gradient of $0.1 \mathrm{~T} / \mathrm{m}$, which is applied using gradient (Anderson) coils, resulting in an one-dimensional spatial resolution of 1 and $4 \mathrm{~mm}$ for $\mathrm{H}$ and $\mathrm{Na}$, respectively. In order to accurately scan the specimen we move the sample through this magnetic field gradient using a step-motor. In this work we use a reference sample that contains an aqueous electrolyte with $4 \mathrm{M} \mathrm{NaCl}$ and $0.04 \mathrm{M} \mathrm{CuCl}_{2}$ to monitor and correct for any unwanted changes in the NMR signal, e.g., due to variations of the NMR equipment. Since the sealing with Teflon tape is not perfect some water can evaporate during the experiments resulting in a uniform drying across the sample, for which we correct by dividing the Na signal by the average water content in the sample.

\section{Results}

In order to see the effect of the applied voltage gradient on the $\mathrm{Na}$ ion profiles, we have measured the $\mathrm{Na}$ concentration across the sample at time intervals of $2 \mathrm{~h}$ with and without an applied voltage gradient between the electrodes. After every $2 \mathrm{~h}$ of desalination treatment the samples were removed from the sample holders and measured in the NMR setup for $45 \mathrm{~min}$. The results for both desalination treatments are presented in Fig. 2. All profiles show some fluctuation in the recorded signal. Since these fluctuations are also present prior to the desalination treatment (at $t=0 \mathrm{~h}$ ), we consider them as experimental noise, which originates from the setup. The results of the measurements without an applied voltage gradient
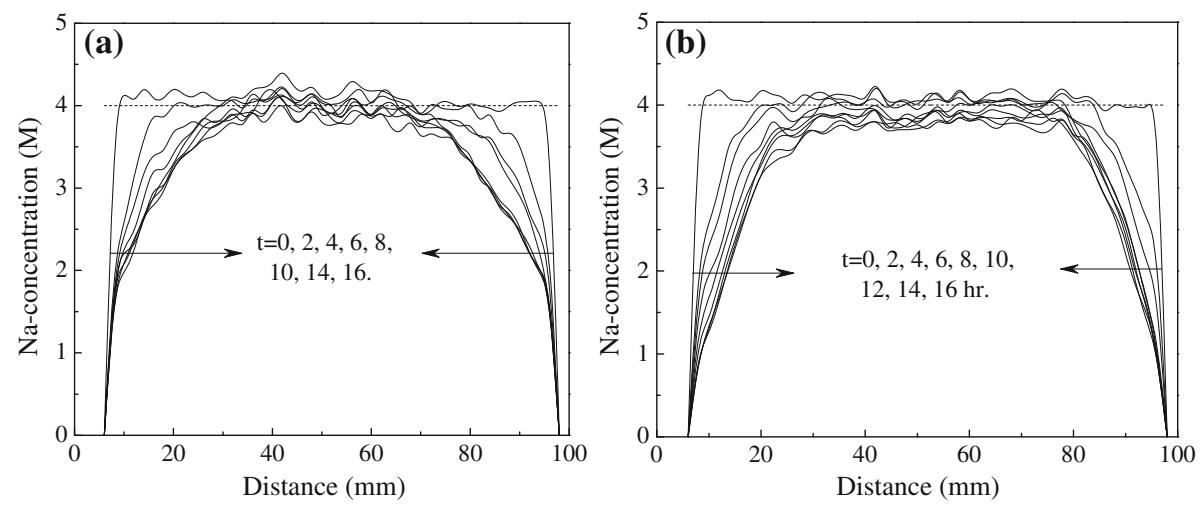

Fig. 2 Na concentration profiles across the porous sample as measured by NMR during a diffusion only, and b by applying 70 Volts between the electrodes. The time interval between the consecutive profiles is $2 \mathrm{~h}$ and the maximum time for each experiment is $16 \mathrm{~h}$ 
are given in Fig. 2a, while the results of the experiments where 70 Volt is applied are presentenced in Fig. 2b. Experiments at different applied voltages (between 35 and 70 Volt) were also performed, and gave consistent results. Therefore, we only discuss the results at 70 Volt. The results show that $\mathrm{Na}$ is removed from the edges of the material with a depleted region that slowly moves into the material. It appears that the applied voltage gradient in Fig. $2 \mathrm{~b}$ does not accelerate the movement of this depletion region significantly. Consequently, the desalination efficiency is nearly identical for both cases, as we will discuss in more detail at the end of this contribution. In addition, the results for both cases show a symmetric behavior. This symmetry arises from the ions to behave as conjugated pairs as discussed in the theory section. The effect of conjugated ions was also experimentally found by Ottosen and Rörig-Dalgaard (2009) who reported that the removal of $\mathrm{NaCl}$ from water-soaked building materials is slower than the removal of $\mathrm{KCl}$. This might be due to the diffusion coefficient of $\mathrm{Na}$, which is lower than that of K (Newman 1973). For systems without ion exchange membranes we found an asymmetric behavior for the ion concentration profiles due to the ingress of protons at the anode and hydroxyl ions at the cathode (Kamran et al. 2012a). Effectively this results in an electrolyte that is enriched with $\mathrm{HCl}$ and $\mathrm{NaOH}$ at the anode and cathode, respectively. Consequently, the profiles for the Na concentration reported in Kamran et al. (2012a) were no longer symmetric.

In order to further check whether the ion exchange membranes indeed block the ingress of $\mathrm{H}$ and $\mathrm{OH}$ ions into the material, we have measured the $\mathrm{pH}$ of the samples after $16 \mathrm{~h}$ of desalination treatment. In order to do so the samples were dry-cut into 8 equal segments, which were ground and the ions were extracted from this material in demi water for at least $24 \mathrm{~h}$. The $\mathrm{pH}$ of these segments was determined by a digital $\mathrm{pH}$ electrode. These measurements revealed that the $\mathrm{pH}$ of only the segments directly adjacent to the sponges and the membranes had changed from initial $\mathrm{pH} \sim 7$ to $\sim 3$ and $\sim 10$ near the anode and cathode, respectively. A large $\mathrm{pH}$ gradient that extends far into the sample as found for systems without any ion exchange membranes was, however, not observed. A similar observation of ion exchange membranes not blocking the ingress of acidic and alkaline regions completely was also reported for soils (Ottosen et al. 2000). However, here the membranes were operating near or above their diffusion limiting current (Ottosen et al. 2000), a condition at which the membrane might partly pass $\mathrm{H}$ and $\mathrm{OH}$ ions (Nikonenko et al. 2010). In our samples the ion concentration adjacent to the membranes at the sample side decreases and reaches zero (see Fig. 2b), so that the dynamics of the system is limited by diffusion, and the diffusion limiting current is reached. This can thus explain why there is some ingress of the acidic and alkaline regions.

In order to measure the electrical field in the material we inserted two platinum wires in the sample spaced $2 \mathrm{~cm}$ apart (see Wada and Umegaki (2001) for a complete description of this procedure) and found an initial electrical field of $30 \mathrm{~V} / \mathrm{m}$. The electrical field decreases as the desalination process proceeds. This is also reflected by the decrease in the current density as function of time as shown in Fig. 3, which is described by $I=5 \times 10^{-4} t\left(\mathrm{~mA} / \mathrm{cm}^{2}\right)$ for $t \approx 0 \longrightarrow 3 \times 10^{4} \mathrm{~s}$ and $I=2(\mathrm{~mA} / \mathrm{cm})^{2}$ for $t>3 \times 10^{4} \mathrm{~s}$.

Using the measured current as a function of time, see Fig 3, we can simulate these measurements using the model described in Sect. 2. We have used the commercially available finite element software package $\mathrm{COMSOL}^{(}{ }^{(}$to do so. We have used this software package because here we can enter partial differential equations, such as Eq. (7), directly into the finite element scheme using the "PDE mode" option. In this software package we have used the standard Backward-Euler method and a direct linear solver to dynamically solve Eq. (7) with a relative and absolute tolerance on the time steps of $10^{-3}$ and $10^{-4}$, respectively. The boundary conditions for Eq. (7) were of the Neumann type according to equation 
Fig. 3 Electrical current recorded during the electrokinetic desalination of the porous brick samples after application of 70 Volts between the electrodes. The line represents the input for the model

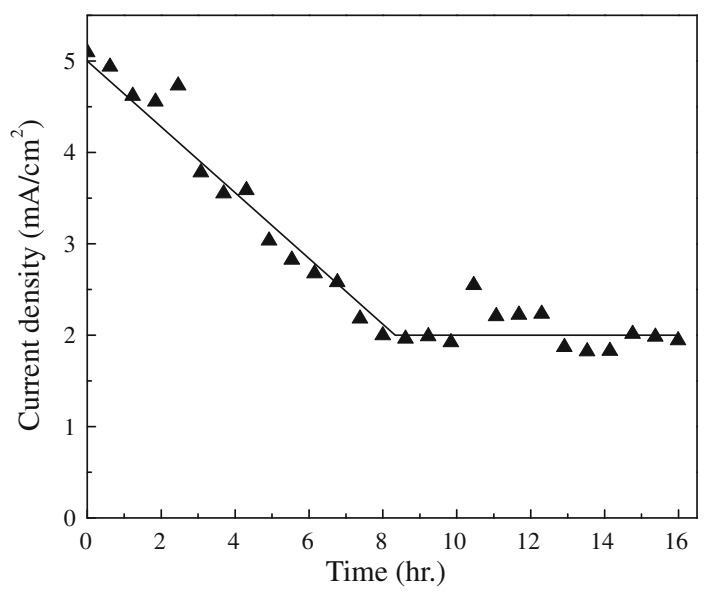

Eq. (9). The values for Eq. (9) were entered using the "functions mode," where we constructed a lookup table for the electrical current as function of time using three points, namely; $I=5 \mathrm{~mA} / \mathrm{cm}^{2}$ at $t=0, I=2 \mathrm{~mA} / \mathrm{cm}^{2}$ at $t=3 \times 10^{4} \mathrm{~s}$, and $I=2 \mathrm{~mA} / \mathrm{cm}^{2}$ at $t=$ $6 \times 10^{4} \mathrm{~s}$, which correspond to Fig. 3. The electrical current, and thus Eq. (9), as function of time is now computed using a linear interpolation between these points. The equations were solved on a one-dimensional geometry of $90 \mathrm{~mm}$ length, which was meshed with a grid containing a maximum element size of $1 \mathrm{~mm}$ and a refinement near the edges toward $0.1 \mathrm{~mm}$ element size with a growth rate factor of 1.1. The electrical field according to Eq. (6) was computed in a separate post-processing step from the results for the dimensionless salt concentration. The input parameters for the diffusion coefficients and the mobility are their values in free water, namely; $D_{\mathrm{Na}}=6.7 \times 10^{-10} \mathrm{~m}^{2} / \mathrm{s}, D_{\mathrm{Cl}}=1 \times 10^{-9} \mathrm{~m}^{2} / \mathrm{s}, \mu_{\mathrm{Na}}=$ $2.6 \times 10^{-8} \mathrm{~m}^{2} /(\mathrm{sV}), \mu_{\mathrm{Cl}}=4 \times 10^{-8} \mathrm{~m}^{2} /(\mathrm{sV})$ (Newman 1973; Atkins and de Paula 2002). By adjusting the tortuosity, $\tau$, the time response of the system can be altered; e.g., a higher value for the tortuosity results in a lower effective ambipolar diffusivity, $D^{*}$, and consequently in a slower response. Here we adjusted the tortuosity in such a way that the simulated time response became equal to the experimental response found previously for the desalination of fired clay brick without membranes (see Kamran et al. 2012a). From this fitting procedure we found that $\tau$ equal to 2 gave excellent agreement between the simulation results and the above-mentioned data. Consequently, the ambipolar diffusivity used in this work becomes $D^{*}=8 \times 10^{-10} \mathrm{~m}^{2} / \mathrm{s}$, which is equal to the ambipolar diffusivity found previously for the desalination of fired clay brick (Kamran et al. 2012a).

The simulation results for the $\mathrm{Na}$ ion concentration profiles are presented in Fig. 4, and show a trend similar to the experimental profiles of Fig. 2b. A clear depletion region at the edge of the material is observed just after applying the electrical current to the edges. However, the salt concentration at the very edge of the material, i.e., at $x=0$ and $x=L$, does not reach zero for prolonged time, but levels off to a value of approximately 0.1 . The value for the salt concentration at these edges will only further decrease for higher values of the current density. In addition to the Na concentration profiles we also computed the evolution of the voltage drop across the system, see Fig. 4b. The calculated initial electrical field is approximately $6 \mathrm{~V} / \mathrm{m}$ and drops steadily to approximately $3 \mathrm{~V} / \mathrm{m}$ after $16 \mathrm{~h}$ of desalination. Within the expected accuracy of all the parameters used, the initial value of the electrical field is of the same order of magnitude as its experimental value of $30 \mathrm{~V} / \mathrm{m}$. For prolonged 

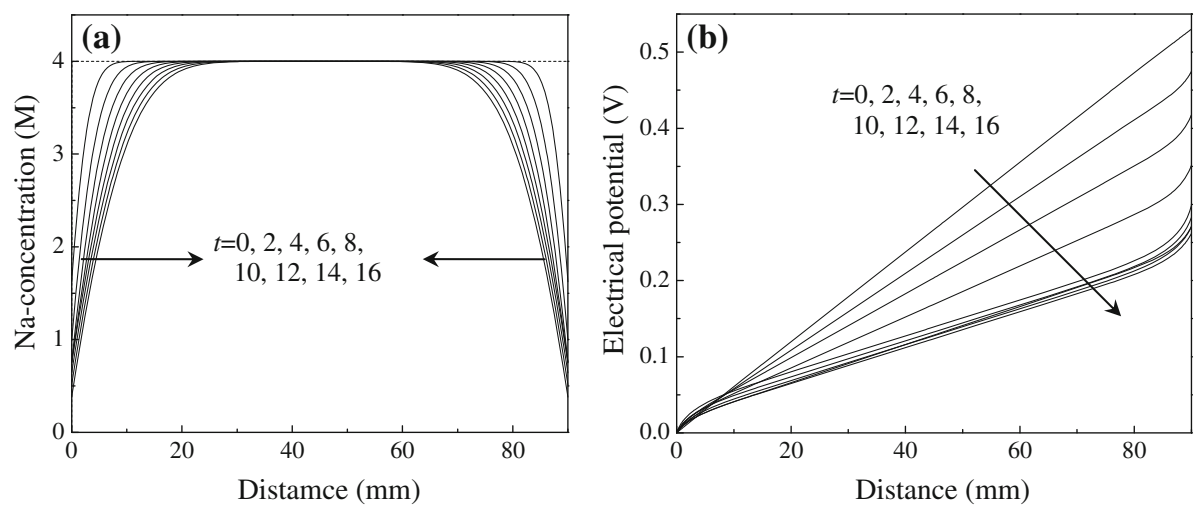

Fig. 4 Simulation results for $\mathbf{a}$ the $\mathrm{Na}$ concentration profiles and $\mathbf{b}$ the voltage across the material as function of time for $D_{\mathrm{Na}}=6.7 \times 10^{-10}, D_{\mathrm{Cl}}=1 \times 10^{-9} \mathrm{~m}^{2} / \mathrm{s}, \mathrm{Na}=2.6 \times 10^{-8}, \mathrm{Cl}=4 \times 10^{-8} \mathrm{~m}^{2} /(\mathrm{sV})$, $L=9 \times 10^{-2} \mathrm{~m}$, and the applied current according to Fig. 3 .

time we can clearly see that the initial linear voltage drop starts to show a non-linear behavior near the edges of the system, where the ions are depleted and thus the conductivity of the electrolyte decreases. As a result of the decreased conductivity, the current through the system will decrease for a constant applied voltage gradient between the electrodes. In a system without membranes acidic and alkaline region will form locally at the anode and cathode, respectively. These regions will thus introduce additional charge carriers (i.e., $\mathrm{H}^{+}$ and $\mathrm{OH}^{-}$ions), which will increase the conductivity. Consequently, the non-linear behavior of the voltage at the edges is reduced in the absence of membranes (see Kamran et al. 2012b). In the simulation results reported here, the electrical field at the very edges of the material increase to a value of approximately $25 \mathrm{~V} / \mathrm{m}$. The value for the electrical field is limited by the finite value for the salt concentration at the edges, which levels off as mentioned above. Consequently, the electrical field cannot grow unbounded.

\section{Discussion}

In order to compare the various desalination methods we can have a look at their efficiency, i.e., the decrease of the total amount of sodium in the sample as function of time. This efficiency is computed by integrating the time-dependent $\mathrm{Na}$ ion concentration profiles and normalize it according to $\eta=\left(1-L^{-1} \int_{0}^{L} C_{\mathrm{Na}} / C_{\mathrm{Na}}^{0} d x\right) \times 100 \%$. We compare four different methods all used on our fired clay brick samples, namely;

1. Electrokinetic desalination without membranes,

2. Desalination by diffusion using poultices,

3. Electrokinetic desalination with membranes,

4. Desalination by diffusion with membranes.

For the electrokinetic experiments without membranes it was found that the $\mathrm{Na}$ ions were expelled in a region near the anode due to the ingress of an acidic region. Although this acidic region might harm the sample, this method achieves the highest efficiency of all processes (see Fig. 5; Table 1). This high efficiency is achieved by the acidic and alkaline regions, which progress into the material, and drive out the $\mathrm{Na}$ and $\mathrm{Cl}$ ions. The final result is that an acidic region containing $\mathrm{HCl}$ and an alkaline region containing $\mathrm{NaOH}$ is obtained. 
Fig. 5 Efficiency of the various desalination processes. The symbols indicate: $(\boldsymbol{\Delta})$ electrokinetic desalination without membranes, $(\boldsymbol{\nabla})$ desalination by diffusion without membranes, $(\bullet)$ electrokinetic desalination with membranes, and ( $\bullet$ ) desalination by diffusion with membranes. The lines are a guide to the eye

Table 1 Steady-state efficiency of the various desalination processes according to Fig. 5.

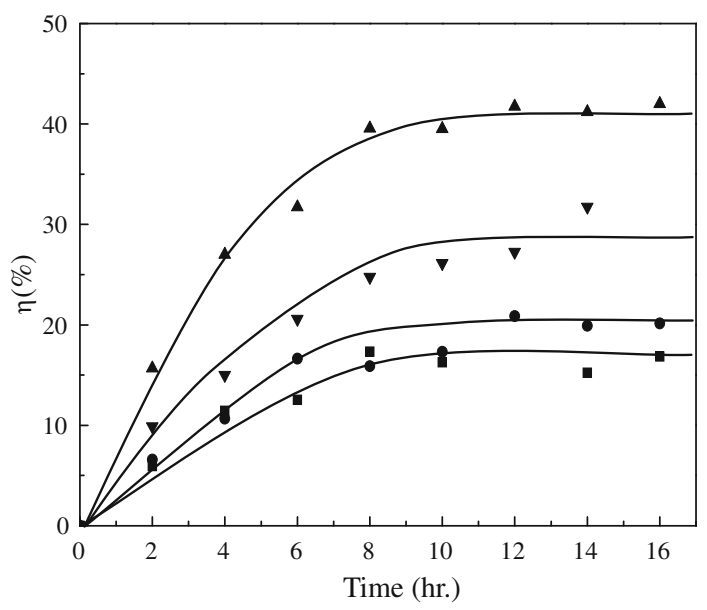

\begin{tabular}{lc}
\hline Desalination treatment & Steady-state efficiency (\%) \\
\hline Electrokinetic w/o membranes & 42 \\
Diffusion using poultices & 32 \\
Electrokinetic with membranes & 20 \\
Diffusion with membranes & 17 \\
\hline
\end{tabular}

The second highest desalination efficiency is achieved by diffusion without any membranes. The difference in efficiency between electrokinetic desalination and desalination by diffusion without any membranes is more significant than for the system with membranes since for the latter case the efficiency for both situations nearly collapse onto one curve. Therefore, we conclude that applying an electrical field across a sample is only beneficial for systems without membranes. In case of membranes, the diffusion limiting current is reached fast and the desalination cannot be accelerated significantly by increasing the electrical field strength. In addition, at currents above the diffusion limit the membranes can lose their barrier function so that acidic and alkaline regions will ingress into the material, as seen for electrokinetic desalination without membranes. These regions can be harmful for building materials.

\section{Conclusions}

From the experimental observations we conclude that during the electrokinetic desalination of porous materials ion exchange membranes can reduce the ingress of acidic and alkaline regions. This is beneficial since these regions can be harmful to construction and building materials. The reduction of the acidic and alkaline regions comes, however, at the cost of the rate of salt removal. Namely, the sodium ion removal rate for electrokinetic desalination without membranes is significantly higher than while using membranes. Moreover, the salt removal rate without any membranes driven by diffusion only is still higher than the electrokinetic desalination with membranes. The reason for this is that the ion exchange membranes provide a penalty for the ions to leave the porous material. The use of these membranes is thus ineffective for the desalination of building materials and should therefore be omitted. 
Finally, we conclude that the experimental data for the sodium ion concentration profiles can be described by a simple Fickian diffusion equation with the measured electrical current as a time-dependent boundary condition.

Acknowledgements We are grateful to the Higher Education Commission (Pakistan) and Nuffic (The Netherlands) for their financial support. M.V.S acknowledges support under project number M81.6.10383 in the framework of the Research Program of the Materials innovation institute in the Netherlands (www.m2i. nl).

\section{References}

Abragam, A.: The Principles of Nuclear Magnetism. Clarendon, Oxford (1961)

Atkins, P., de Paula, J.: Atkins Physical Chemistry. Oxford University Press, London (2002)

Bard, A.J., Faulkner, L.R.: Electrochemical methods. Wiley, New York (2001)

Bazant, M.Z., Thorton, K., Ajdari, A.: Diffuse-charge dynamics in electrochemical systems. Phys. Rev. E 70, 021506 (2004)

Bazant, M.Z., Chu, K.T., Bayly, B.J.: Current-voltage relations for electrochemical thin films. SIAM J. Appl. Math. 65, 1463-1484 (2005)

Beddoe, R.E., Dorner, H.W.: Modelling acid attack on concrete: Part I. The essential mechanisms. Cem. Concr. Res. 35, 2333-2339 (2005)

Biesheuvel, P.M., van der Wal, A.: Membrane capacitive deionization. J. Membrane Sci. 346, 256-262 (2010)

Biesheuvel, P.M., Franco, A.A., Bazant, M.Z.: Diffuse charge effects in fuel cell membranes. J. Electrochem. Soc. 156, B225-B233 (2009a)

Biesheuvel, P.M., van Soestbergen, M., Bazant, M.Z.: Imposed currents in galvanic cells. Electrochim Acta 54, 4857-4871 (2009b)

Bonnefont, A., Argoul, F., Bazant, M.Z.: Analysis of diffuse-layer effects on time-dependent interfacial kinetics. J. Electroanal. Chem. 500, 52-61 (2001)

Chu, K.T., Bazant, M.Z.: Electrochemical thin films at and above the classical limiting current. SIAM J. Appl. Math. 65, 1485-1505 (2005)

Coussy, O., Eymard, R.: Non-linear binding and the diffusion-migration test. Trans. Porous. Med. 53, 51-57 (2003)

Crank, J.: The Mathematics of Diffusion. Clarendon, Oxford (1990)

Deen, W.M.: Analysis of Transport Phenomena. Oxford University Press, Oxford, (1998)

Dlugolecki, P., Nymeijer, K., Metz, S., Wesseling, M.: Current status of ion exchange membranes for power generation from salinity gradients. J. Membrane Sci. 319, 214-222 (2008)

Farmer, J.C., Fix, D.V., Mack, G.V., Pekala, R.W., Poco, J.F.: Capacitive deionization of $\mathrm{NaCl}$ and $\mathrm{NaNO}_{3}$ Solutions with Carbon Aerogel Electrodes. J. Electrochem. Soc. 143, 159-169 (1996)

Hahn, E.L.: Spin Echoes. Phys. Rev. 80, 580-594 (1950)

Jacobs, R.A., Sengun, M.Z., Hicks, R.E., Probstein, R.F.: Model and experiments on soil remediation by electric fields. J. Environ. Sci. Health A29, 1933-1955 (1994)

Johnson, A.M., Newman, J.: Desalting by means of porous carbon electrodes. J. Electrochem. Soc. 118, 510-517 (1971)

Kamran, K., Pel, L., Sawdy, A., Huinink, H.P., Kopinga, K.: Desalination of porous building materials by electrokinetics: an NMR study. Mater. Struct. 45, 297-308 (2012a)

Kamran, K., van Soestbergen, M., Huinink, H.P., Pel, L.: Inhibition of electrokinetic ion transport in porous materials due to potential drops induced by electrolysis. Electrochimica Acta 78, 229-235 (2012b)

Kim, S., Kim, K.: Monitoring of electrokinetic removal of heavy metals in tailing-soils using sequential extraction analysis. J. Hazard. Mater. B85, 195-211 (2001)

Levich, V.G.: Physicochemical Hydrodynamics. Prentice-Hall, Englewood Cliffs, p. 281 (1962)

Lu, X., Viljanen, M.: Determination of salt diffusion coefficient in brick: analytical methods. Trans. Porous. Med. 49, 241-246 (2002)

Newman, J.S.: Electrochemical Systems. Prentice-Hall, Englewood Cliffs (1973)

Nikonenko, V.V., Pismenskaya, N.D., Belova, N.D., Sistat, P., Huguet, P., Pourcelly, P., Larchet, C.: Intensive current transfer in membrane systems: Modelling, mechanisms and application in electrodialysis. Adv. Coll. Int. Sci. 160, 101-123 (2010)

Nystrøm, G.M., Ottosen, L.M., Villumsen, A.: Test of experimental set-ups for electrodialytic removal of $\mathrm{Cu}$, $\mathrm{Zn}, \mathrm{Pb}$ and $\mathrm{Cd}$ from different contaminated harbour sediments. Eng. Geol. 77, 349-357 (2005) 
Ottosen, L.M., Rörig-Dalgaard, I.: Desalination of a brick by application of an electric DC field. Mater Struct. 42, 961-971 (2009)

Ottosen, L.M., Hansen, H.K., Hansen, C.B.: Water splitting at ion-exchange membranes and potential differences in soil during electrodialytic soil remediation. J. App. Electrochem. 30, 1199-1207 (2000)

Pel, L., Kopinga, K., Kaasschieter, E.F.: Saline absorption in calcium-silicate brick observed by NMR scanning. J. Phys. D: Appl. Phys. 33, 1380-1385 (2000)

Porada, S., Sales, B.B., Hamelers, H.V.M., Biesheuvel, P.M.: Water desalination with wires. Phys. Chem. Lett. 3, 1613-1618 (2012)

Prieve, D.C.: Changes in zeta potential caused by a dc electric current for thin double layers. Colloids Surf. A 250, 67-77 (2004)

Probstein, R.F., Hicks, R.E.: Removal of contaminants from soils by electric fields. Science 260, 298 (1993)

Ribeiro, A.B., Mexia, J.T.: A dynamic model for the electrokinetic removal of copper from a polluted soil. J. Hazard. Mater. 56, 257-271 (1997)

Sghaier, N., Prat, M., Nasrallah, S.B.: On ions transport during drying in a porous medium. Trans. Porous. Med. 67, 243-274 (2007)

Smyrl, W.H., Newman, J.: Double layer structure at the limiting current. Trans. Faraday Soc. 63, 207-216 (1967)

van Soestbergen, M.: Diffuse layer effects on the current in galvanic cells containing supporting electrolyte. Electrochimica Acta 55, 1848-1854 (2010)

van Soestbergen, M., Biesheuvel, P.M., Bazant, M.Z.: Diffuse-charge effects on the transient response of electrochemical cells. Phys. Rev. E 81, 021503 (2010)

Vetter, K.J.: Electrochemical Kinetics. Academic Press, New York (1967)

Viadero, R.C., Reed, B.E., Berg, M., Ramsey, : A Laboratory-scale study of applied voltage on the electrokinetic separation of lead from soils. J. Separation Sci. Tech. 33, 1833-1859 (1998)

Wada, S.I., Umegaki, Y.: Major ion and electrical potential distribution in soil under electrokinetic remediation. Environ. Sci. Technol. 36, 2151-2155 (2001)

Yang, G.C.C., Lin, S.: Removal of lead from a silt loam soil by electrokinetic remediation. J. Hazard. Mater. 58, 285-299 (1998)

Yeung, A.T., Gu, Y.: A review on techniques to enhance electrochemical remediation of contaminated soils. J. Hazard. Mater. 195, 11-29 (2001)

Zhao, R., Biesheuvel, P.M., Miedema, H., Bruning, H., van der Wal, A.J.: Charge efficiency: a functional tool to probe the double-layer structure inside of porous electrodes and application in the modeling of capacitive deionization. Phys. Chem. Lett. 1, 205-210 (2010) 